\title{
The Chaplygin sleigh with friction moving due to periodic oscillations of an internal mass
}

\author{
Ivan A. Bizyaev • Alexey V. Borisov - Sergey P. Kuznetsov
}

Received: date / Accepted: date

\begin{abstract}
For a Chaplygin sleigh moving in the presence of weak friction, we present and investigate two mechanisms of arising acceleration due to oscillations of an internal mass. In certain parameter regions, the mechanism induced by small oscillations determines acceleration which is on average one-directional. The role of friction is that the velocity reached in the process of the acceleration is stabilized at a certain level. The second mechanism is due to the effect of parametric excitation of oscillations, when the internal moving particle is comparable in mass with the main platform, and, as occurs, a necessary condition for the acceleration is presence of friction. The parametric instability and the resulting acceleration of the sleigh turn out to be bounded if the line of oscillations of the moving mass is displaced from the center of mass. The steady-state regime of motion is in many cases associated with a chaotic attractor; accordingly, the motion of the sleigh turns out to be similar to the process of random walk on a plane.
\end{abstract}

Russian Science Foundation, Grant no. 15-12-20035

Ivan A. Bizyaev

Udmurt State University, Universitetskay 1, Izhevsk, 426034,

Russian Federation

E-mail: bizaev_90@mail.ru

Alexey V. Borisov

Udmurt State University, Universitetskay 1, Izhevsk, 426034,

Russian Federation

E-mail: borisov@rcd.ru

Sergey P. Kuznetsov (corresponding author)

Udmurt State University, Universitetskay 1, Izhevsk, 426034,

Russian Federation,

Kotel'nikov's Institute of Radio-Engineering and Electronics of RAS, Saratov Branch, Zelenaya 38, Saratov, Russian Federation,

E-mail: spkuz@yandex.ru
Keywords Nonholonomic mechanics · Chaplygin sleigh - parametric oscillator - strange attractor . Lyapunov exponent · chaotic dynamics

\section{Introduction}

Nonholonomic mechanical systems, where algebraic relations (constraints) imposed on dynamical variables are given by equations involving generalized velocities, arise in the context of many problems of practical importance, for example, in considering mobile devices, and have been a subject of many classical studies [1, 2, 3, 4,5, 6. With basic definitions, historical aspects and the modern state of the theory of nonholonomic dynamical systems the reader can get acquainted with the review article [7. Recent developments in robotics have given a new impetus to this area of research in mechanics. Nonholonomic systems serve as simple models, which are attractive as compared with alternative methods of description, which require a detailed analysis of friction effects, and provide insight on main properties of mechanical motions delivering efficient tools, particularly, for developing motion control algorithms [6, 8, 9, 10, 11.

The class of nonholonomic systems is very wide and is characterized by a hierarchy of dynamical behaviors ranging from regular motions of integrable systems to complex dynamics of nonintegrable systems, depending on a number of invariants and symmetries inherent in the problem [12. In particular, systems with the above-mentioned complex dynamics include the rattleback problem concerned with motions of a rigid body with a convex smooth surface on a rough plane (when the principal axes determining the geometric properties of the body and those determining the dynamical properties are different). In such systems, mechanical energy 
is conserved, but there is no preservation of phase volume, so that in the course of time evolution an element of the volume can undergo, depending on its location, either compression or extension. For this reason, they can exhibit phenomena which might seem to be specific only to dissipative dynamics, like attractive fixed points, limit cycles, and even strange attractors [13,14, 15.

The Chaplygin sleigh [16,17] is a well-known and well-established paradigmatic model of nonholonomic mechanics. The Chaplygin sleigh is a rigid body - a platform moving on a horizontal plane under the condition that the translational velocity at some point of the sleigh is always oriented along a certain direction fixed relative to the platform. This condition is a nonholonomic constraint which is imposed on the system and which can be implemented by means of a knife edge (skate) fastened to the sleigh or by means of a wheel pair [18. A detailed analytical study of the problem of the sleigh motion using explicit quadratures was carried out in the 1930s by Carathéodory [17. Depending on the position of the center of mass relative to the knife edge, the sleigh asymptotically tends to a rectilinear motion or to a circular motion. It is interesting that the dynamics of the Chaplygin sleigh on an inclined plane is not integrable and exhibits random-like asymptotic behavior depending on initial conditions [19].

Various generalizations of the problem of the Chaplygin sleigh were considered by many authors $[20,21,22$, 23, 24, 25, 26, 27, 28. In particular, attention was given to problems of the motion of the sleigh in the presence of periodic impulse impacts [20,21], with periodic switchovers of the nonholonomic constraint to different locations [23,24], and with periodic transfers of the massive load placed on the sleigh [25,26]. Analysis was also made of the motion of the sleigh under the action of random forces which model a fluctuating continuous medium [28, when, according to the authors, the motion resembles random walks of bacterial cells with a diffusion component.

The study of the possibility of directed motion on a plane or in a medium induced by the presence of internal moving masses $[8,29,9,10,30,31,22$ is of fundamental importance from the viewpoint of robotics. In particular, such problems are obviously interesting and insightful with respect to objects that are described in terms of models of nonholonomic mechanics.

In [25], general equations are formulated which describe the motion of the Chaplygin sleigh with a given periodic transfer of several internal point masses relative to the platform. It is shown that, depending on the parameters, the system exhibits different types of motions, including those corresponding to strange attrac- tors, with which chaotic (diffusion) trajectories of the sleigh on a plane are associated. This is interpreted as a nonholonomic analog of the well-known phenomenon of Fermi's acceleration [32,33.

In [26], we considered a special case of the Chaplygin sleigh moving due to a given periodic motion of a single internal point mass perpendicularly to the axis passing through the center of mass of the platform and through the point of application of the nonholonomic constraint. We showed a possibility of unbounded acceleration of regular directed motion of the sleigh due to small oscillations of the internal mass, so that the longitudinal momentum of the sleigh increases asymptotically, in proportion to time to the $1 / 3$ power. Using numerical simulation, we also revealed and demonstrated periodic, quasi-periodic and chaotic motions, which are related to attractors corresponding to bounded variations in the velocity. Since the equations of the system are invariant under time reversal, the presence of attractors which correspond to the observed steady motions implies the presence of repellers which can be found when tracking the dynamics in backward time.

This paper, which is a further development of [26], is devoted to the study of the motion of the Chaplygin sleigh, induced by periodic oscillations of an internal mass, in the presence of a weak friction. In Section 1 we formulate basic equations of a mathematical model. In Section 2 we analyze the mechanism of acceleration that takes place under small oscillations of the internal mass and leads to regular, on average unidirectional motion of the sleigh; in this case the role of friction is to stabilize the velocity of motion at a fixed level - the smaller the friction, the higher the velocity. Section 3 is concerned with the other mechanism of acceleration due to the effect of parametric excitation of oscillations in a situation where the oscillating mass constitutes a major portion of the mass of the platform. In the case of motion of the internal mass in a straight line passing through the center of mass, the problem reduces to a linear equation with periodic coefficients, the increase in parametric oscillations turns out to be unbounded and the linear dissipation (viscous friction) does not lead to saturation. Parametric instability, and thus the degree of acceleration, of the sleigh are bounded if the line of oscillations of the internal mass is displaced from the center of mass. The steady-state regime of motion corresponds to attractors of the reduced system of equations, which in many cases are chaotic; accordingly, the motion of the sleigh undergoing acceleration is also chaotic and similar to a diffusion process. Section 4 discusses differences between the patterns of motions associated with chaotic attractors in the nonholonomic model without friction 
and in the model with friction. In the Conclusion (Section 5) we summarize and discuss the results of this research.

\section{Basic equations}

We consider a mechanical system, the sleigh, the basis of which is a platform capable of sliding without friction on a horizontal plane with a constraint supplied at some point $R$ restricted to move exclusively in a certain direction fixed relative to the platform that can be thought as direction of the attached "knife-edge" (Fig.1). Additionally, a material point of mass $m_{p}$ is placed on the sleigh that performs a predetermined oscillatory motion on it.

Let $u_{1}, u_{2}$ be the projections of the velocity of the point $R$ measured in a laboratory coordinate system $O X Y$ onto the axes of a moving reference frame $R \xi \eta$, and let $\omega$ be the angular velocity of the platform. The kinetic energy of the platform, together with a moving material point which has coordinates $\xi_{p}(t), \eta_{p}(t)$ in the reference frame attached to the platform, is

$T=\frac{1}{2} m_{0} u_{1}^{2}+\frac{1}{2} m_{0}\left(u_{2}+a \omega\right)^{2}+\frac{1}{2} J_{0} \omega^{2}$

$+\frac{1}{2} m_{p}\left(u_{1}+\dot{\xi}_{p}-\eta_{p} \omega\right)^{2}+\frac{1}{2} m_{p}\left(u_{2}+\dot{\eta}_{p}+\xi_{p} \omega\right)^{2}$.

Here, $m_{0}$ is the mass of the platform, $m_{p}$ is the mass of the particle, and $J_{0}$ is the moment of inertia of the platform relative to its center of mass. Let

$R=\frac{1}{2} c_{1} u_{1}^{2}+\frac{1}{2} c_{2} u_{2}^{2}+\frac{1}{2} c_{3} \omega^{2}$

be the dissipative Rayleigh function, where the coefficients $c_{1,2}$ are responsible for friction along the axes $\xi$ and $\eta$, and the coefficient $c_{3}$, for friction with respect to the rotational motion.

Using these relations, we write the Lagrange equations

$$
\begin{aligned}
& \frac{d}{d t}\left(\frac{\partial T}{\partial u_{1}}\right)=\omega \frac{\partial T}{\partial u_{2}}-\frac{\partial R}{\partial u_{1}}, \\
& \frac{d}{d t}\left(\frac{\partial T}{\partial u_{2}}\right)=-\omega \frac{\partial T}{\partial u_{1}}+\lambda-\frac{\partial R}{\partial u_{2}}, \\
& \frac{d}{d t}\left(\frac{\partial T}{\partial \omega}\right)=u_{2} \frac{\partial T}{\partial u_{1}}-u_{1} \frac{\partial T}{\partial u_{2}}-\frac{\partial R}{\partial \omega},
\end{aligned}
$$

where $\lambda$ is a Lagrange multiplier defined to ensure the imposed non-holonomic constraint condition $u_{2}=0$. (In fact, it is sufficient simply to omit the second relation since $\lambda$ does not enter the remaining equations.)

Introducing the generalized momenta

$$
\begin{aligned}
P=\left.\frac{\partial T}{\partial u_{1}}\right|_{v_{2}=0}= & m_{0} u_{1}+m_{p}\left(u_{1}+\dot{\xi}_{p}-\eta_{p} \omega\right), \\
M=\left.\frac{\partial T}{\partial \omega}\right|_{v_{2}=0}= & \left(m_{0} a^{2}+J_{0}+m_{p}\left(\xi_{p}^{2}+\eta_{p}^{2}\right)\right) \omega \\
& -m_{p} u_{1} \eta_{p}+m_{p}\left(\xi_{p} \dot{\eta}_{p}-\dot{\xi}_{p} \eta_{p}\right)
\end{aligned}
$$

and assuming $m=m_{0}+m_{p}, \mu=m_{p} / m, I_{0}=J_{0} / m$, we rewrite the equations (3) as

$$
\begin{aligned}
& \dot{P}=m\left\{a \omega+\mu\left[\dot{\eta}_{p}+\left(\xi_{p}-a\right) \omega\right]\right\} \omega-c_{1} u_{1}, \\
& \dot{M}=-m\left\{a \omega+\mu\left[\dot{\eta}_{p}+\left(\xi_{p}-a\right) \omega\right]\right\} u_{1}-c_{3} \omega .
\end{aligned}
$$

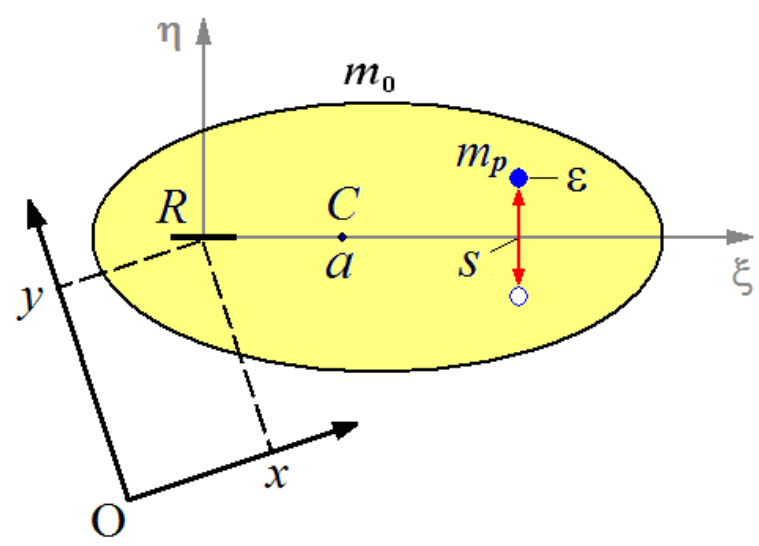

Fig. 1 Symbols and coordinate systems for the Chaplygin sleigh with the oscillating internal mass $m_{p}$. The other symbols are: $m_{0}$ is the mass of the platform, $R$ is the point of application of the nonholonomic constraint (the position of the knife edge), and $\mathrm{C}$ is the center of mass of the platform. $\mathrm{O} x y$ is the laboratory reference frame, and $R \xi \eta$ is the coordinate system attached to the platform.

The quantities $\omega$ and $u_{1}$, which appear here on the right-hand sides, are expressed in terms of $P$ and $M$ algebraically by the set of linear equations

$$
\begin{gathered}
u_{1}+\mu\left(\dot{\xi}_{p}-\eta_{p} \omega\right)=P / m, \\
{\left[(1-\mu) a^{2}+I_{0}+\mu\left(\xi_{p}^{2}+\eta_{p}^{2}\right)\right] \omega-\mu \eta_{p} u_{1}} \\
=M / m-\mu\left(\xi_{p} \dot{\eta}_{p}-\dot{\xi}_{p} \eta_{p}\right) .
\end{gathered}
$$

Let $\xi_{p}=s, \eta_{p}=\varepsilon \sin \Omega t$. Using the dimensionless time $\tau=\Omega t$ and the normalized variables $p=P / m \Omega$, $q=M / m \Omega, u=u_{1} / \Omega, w=\omega / \Omega$, we obtain

$\dot{p}=(D w+\mu \varepsilon \cos \tau) w-\nu_{1} u$,

$\dot{q}=-(D w+\mu \varepsilon \cos \tau) u-\nu_{3} w$,

where $D=a-\mu a+\mu s, \nu_{1}=c_{1} m^{-1}, \nu_{3}=c_{3} m^{-1}$. Now the dots denote derivatives with respect to the dimensionless time $\tau$, and the formulae of constraints of the generalized velocities and momenta take the form

$u-w \mu \varepsilon \sin \tau=p$,

$-u \mu \varepsilon \sin \tau+\left(J+\mu \varepsilon^{2} \sin ^{2} \tau\right) w=q-\mu s \varepsilon \cos \tau$,

where $J=I_{0}+a^{2}+\mu\left(s^{2}-a^{2}\right)$. It is easy to verify that the equations are invariant under the change of variables

$\tau \rightarrow \tau+\pi, q \rightarrow-q, p \rightarrow p, w \rightarrow-w, u \rightarrow u$.

Equations (7) and (8), which govern the evolution of two variables $p, q$ in continuous time, define the reduced system, which can be considered independently of the other variables relating to configuration space. These variables, namely, the coordinates of the point where the knife edge $R$ is located in the laboratory reference frame $x, y$ and the angle of rotation of the platform $\phi$, are governed by the equations

$\dot{x}=u \cos \varphi, \dot{y}=u \sin \varphi, \dot{\varphi}=\omega$. 
This completes the mathematical formulation of the problem.

When analyzing the sleigh movements, information on the reaction force acting at the point of application of the nonholonomic constraint perpendicular to the "knife edge" may be useful. It is given just by the Lagrange multiplier in the second equation (3) and, as can be shown, in the normalized form, is determined by the expression

$F=\lambda / m \Omega^{2}=u w-\varepsilon \mu\left(1+w^{2}\right) \sin \tau+(a-\mu a+\mu s) \dot{w}$.

Suppose that the values $p_{n}, q_{n}$ of the variables are assigned at time $\tau=2 \pi n$. Solving numerically the equations on the time interval $\Delta \tau=2 \pi$ under these initial conditions, we can obtain the new values

$\left(p_{n+1}, q_{n+1}\right)=\mathbf{f}\left(p_{n}, q_{n}\right)$.

Thus we have defined a two-dimensional stroboscopic Poincaré map, which is convenient to describe and represent the results of analysis of the dynamical behavior of the reduced system in discrete time.

The change of variable $q=z-p \mu \varepsilon \sin \tau$ brings the reduced equations to an equivalent form, which is sometimes more convenient,

$\dot{p}=(D w+\mu \varepsilon \cos \tau) w-\nu_{1} u$,

$\dot{z}=-\left(D p+\nu_{3}\right) w-\nu_{1} u \mu \varepsilon \sin \tau$,

$w=\frac{z-\mu s \varepsilon \cos \tau}{J+\mu(1-\mu) \varepsilon^{2} \sin ^{2} \tau}, u=p+w \mu \varepsilon \sin \tau$.

We note that in the case $\nu_{1}=0$ the terms containing the coefficient $\nu_{3}$ are eliminated by a variable change $p=p^{\prime}-\nu_{3} D^{-1}$; therefore, at small friction, the rotational component of friction is, generally speaking, of little importance.

\section{Acceleration under small oscillations}

Consider the motion of the sleigh in a case of small amplitude of oscillations of a small internal mass using an analytical method inspired by 34] and developed for this specific problem in 26]. Under the assumption that the longitudinal momentum $p$ does not vary much over a period of oscillations of the internal mass, we write for it an equation averaged over a period:

$\dot{p}=D \overline{w^{2}}+\mu \varepsilon \overline{w \cos \tau}-\nu_{1} \bar{u}$.

To find the averaged terms appearing on the righthand side of this expression, we neglect the quantity $\varepsilon^{2}$ and, according to the third equation of (13), we set $w=$ $(z-\mu s \varepsilon \cos \tau) J^{-1}, u=p+J^{-1} \mu \varepsilon(z-\mu s \varepsilon \cos \tau) \sin \tau$. Substituting into the second equation of (13) then gives

$\dot{z}=J^{-1}\left(D p+\nu_{3}\right)(-z+\mu s \varepsilon \cos \tau)-\nu_{1} p \mu \varepsilon \sin \tau$, and in the approximation of constancy of $p$ the solution has the form

$$
\begin{aligned}
z= & \mu \varepsilon \frac{\left(D p+\nu_{3}\right)^{2} s+\nu_{1} p J^{2}}{J^{2}+\left(D p+\nu_{3}\right)^{2}} \cos \tau \\
& +\mu \varepsilon J \frac{\left(D p+\nu_{3}\right)\left(s-\nu_{1} p\right)}{J^{2}+\left(D p+\nu_{3}\right)^{2}} \sin \tau .
\end{aligned}
$$

It yields

$$
\begin{aligned}
& w=(z-\mu s \varepsilon \cos \tau) J^{-1} \\
& \quad=\mu \varepsilon \frac{\left(\nu_{1} p-s\right)\left(J \cos \tau-\left(D p+\nu_{3}\right) \sin \tau\right)}{J^{2}+\left(D p+\nu_{3}\right)^{2}},
\end{aligned}
$$

so that

$\overline{w^{2}}=\frac{1}{2} \mu^{2} \varepsilon^{2} \frac{\left(s-\nu_{1} p\right)^{2}}{J^{2}+\left(D p+\nu_{3}\right)^{2}}$,

$\bar{u}=p+\mu \varepsilon \overline{w \sin \tau}=p+\frac{1}{2} \mu^{2} \varepsilon^{2} \frac{\left(D p+\nu_{3}\right)\left(s-\nu_{1} p\right)}{J^{2}+\left(D p+\nu_{3}\right)^{2}}$,

$\overline{w \cos \tau}=\frac{1}{2} \mu \varepsilon \frac{J\left(\nu_{1} p-s\right)}{J^{2}+\left(D p+\nu_{3}\right)^{2}}$.

Thus, equation (14) takes the form

$\dot{p}+\nu_{1} p=$

$\frac{1}{2} \mu^{2} \varepsilon^{2}\left(s-\nu_{1} p\right) \frac{D s-J-\left(2 D p+\nu_{3}\right) \nu_{1}}{J^{2}+\left(D p+\nu_{3}\right)^{2}}$.

According to [26, under the conditions

$D=a-\mu a+\mu s>0$,

$s(D s-J)=s\left(a(s-a)(1-\mu)-I_{0}\right)>0$,

in the absence of friction $\left(\nu_{1}=\nu_{3}=0\right)$ unbounded acceleration occurs with an increase in the momentum of the sleigh. Indeed, integrating equation (19) in this case gives $\frac{1}{3} p^{3} D^{2}+J^{2} p=\frac{1}{2} \mu^{2} s \varepsilon^{2}(D s-J) \tau+$ const, and, since in the region of a large momentum the first term on the left-hand side dominates, the increase in the momentum (and of the velocity of the sleigh in the direction of the knife edge) asymptotically follows the power law $t^{1 / 3}$. In 26, a good agreement is demonstrated between analytical results and those of numerical simulation of acceleration in the absence of friction.

When friction is taken into account, the increase in the velocity ceases to be unbounded and is stabilized at some level. This is illustrated in Fig. 2, where for some specific parameters satisfying conditions (20) the longitudinal momentum is shown versus time according to the results of numerical solution of equations (13) (lines) and the averaged equation (19) with zero initial conditions (dots). It can be seen that the agreement is very good.

Figure 3 shows the plane of the parameters $s$ (the coordinate of the oscillating mass relative to the knife edge) and $\mu$ (the ratio of the oscillating mass to the total mass of the system). White denotes regions where conditions (20) are satisfied, and inside these regions one can see level lines (indicated by corresponding numbers) for the momentum values the sleigh reaches within a prolonged time interval in the presence of friction. 


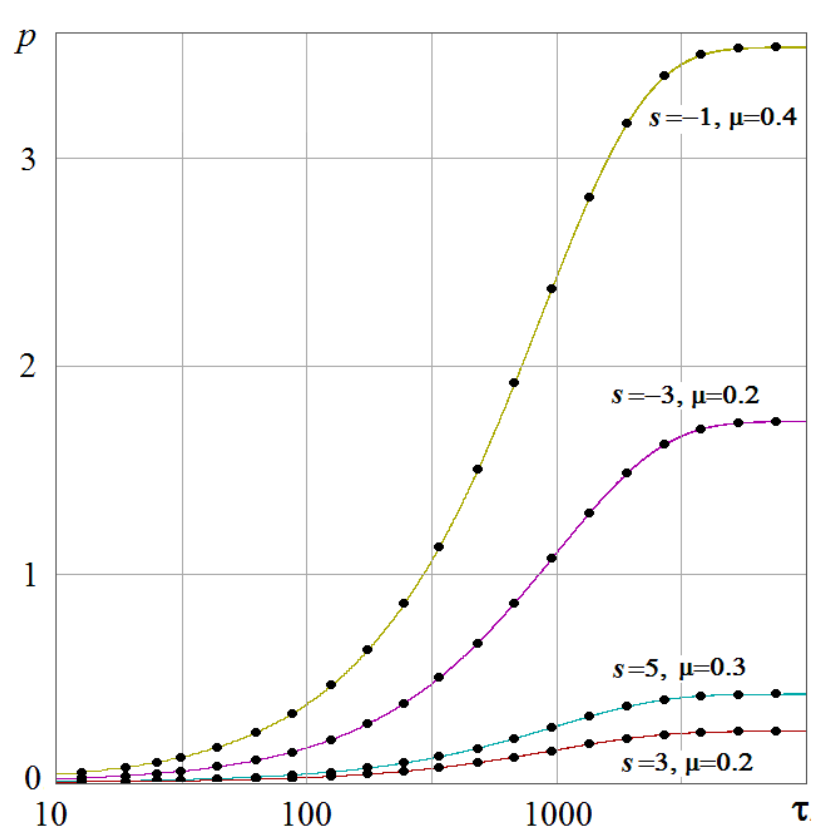

Fig. 2 Dimensionless longitudinal momentum $p$ versus dimensionless time in regions of accelerated motion of the sleigh for different values of $s$ and $\mu$ as obtained from numerical solution of the equations (13) (solid lines) and from the shortened equation (19) (dots). The other parameters are $I_{0}=1, a=1$, $\varepsilon=0.3, \nu_{1}=0.001, \nu_{3}=0$

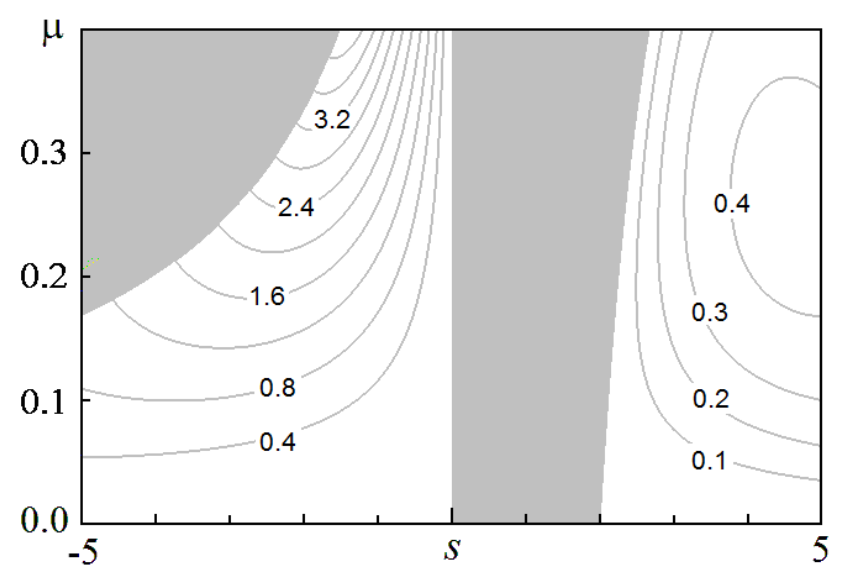

Fig. 3 Regions of acceleration of the sleigh (white) and regions of oscillatory variations in the velocity (grey) on the plane of parameters $s$ and $\mu$. The other parameters are $I_{0}=1$, $a=1, \varepsilon=0.3, \nu_{1}=0.001, \nu_{3}=0$. In the region of acceleration, one can see level lines of the function of the longitudinal momentum values, which the sleigh reaches starting from a rest within a fairly large time interval $\left(10^{3}\right.$ periods of oscillations of the internal mass).

We now will estimate the asymptotic characteristics of the motion of the sleigh. For simplicity we assume that $\nu_{3}=0$, so that the equation takes the form

$\dot{p}=-\nu_{1} p+\frac{1}{2} \mu^{2} \varepsilon^{2}\left(s-\nu_{1} p\right) \frac{D s-J-2 D p \nu_{1}}{J^{2}+D^{2} p^{2}}$.

If $\mu^{2} \varepsilon^{2}<<1$, then, as the sleigh approaches steady motion, which exists under conditions (20) and corre- sponds to $\nu_{1} p \sim \mu^{2} \varepsilon^{2}$, one can neglect terms of higher order in the small parameter and write

$\dot{p}=-\nu_{1} p+\frac{1}{2} \mu^{2} \varepsilon^{2} s \frac{D s-J}{J^{2}+D^{2} p^{2}}$.

Thus, for the steady motion we have

$p J^{2}+D^{2} p^{3}=\frac{1}{2} \mu^{2} \varepsilon^{2} s(D s-J) \nu_{1}^{-1}$.

As long as the parameter $\nu_{1}$ is not very small, the first term on the left-hand side of (23) dominates, and the momentum reached during acceleration grows with decreasing coefficient of friction as a minus first power: $p \sim \frac{1}{2} \mu^{2} \varepsilon^{2} s J^{-2}(D s-J) \nu_{1}^{-1}$. On the other hand, when $\nu_{1}$ is very small, the second term on the left-hand side in (23) dominates, and the momentum reached is inversely proportional to the cubic root of the friction coefficient: $p \sim\left(\frac{1}{2} \mu^{2} \varepsilon^{2} s D^{-2}(D s-J)\right)^{1 / 3} \nu_{1}^{-1 / 3}$. The crossover from one type of dependence to another takes place near the value of the parameter $\nu_{1} \sim \mu^{2} \varepsilon^{2} s(D s-J) D J^{-3}$.

\section{Parametric mechanism of acceleration}

Consider a particular case of equations (13) where the oscillating particle moves in a straight line passing through the center of mass of the whole system (platform plus particle). In this case, $D=0$, and the problem reduces to a system of linear equations with variable coefficients $\dot{p}=\mu \varepsilon w \cos \tau-\nu_{1} u$,

$\dot{z}=-\nu_{3} w-\nu_{1} u \mu \varepsilon \sin \tau$,

$w=\frac{z-\mu s \varepsilon \cos \tau}{J+\mu(1-\mu) \varepsilon^{2} \sin ^{2} \tau}$,

$u=p+w \mu \varepsilon \sin \tau$,

which is similar in structure to equations used in the linear theory of parametric oscillations, such as the Mathieu equation. We note that the presence of dissipation appears to be a necessary condition for the effect of increasing parametric oscillations, since when there is absolutely no friction $\left(\nu_{1}=\nu_{3}=0\right)$, the second equation degenerates to the trivial relation $\dot{z}=0$, whence it follows that $z=$ const.

For each period of the coefficient oscillations, the transformation of the state is defined by a monodromy matrix, which can be found by numerically integrating the homogeneous equations that correspond to the system (24)

$\dot{p}=\mu \varepsilon w \cos \tau-\nu_{1} u$,

$\dot{z}=-\nu_{3} w-\nu_{1} u \mu \varepsilon \sin \tau$,

$w=z /\left[J+\mu(1-\mu) \varepsilon^{2} \sin ^{2} \tau\right]$,

$u=p+w \mu \varepsilon \sin \tau$.

in one period of oscillations with initial conditions (1, $0)$ and $(0,1)$, which gives, respectively, the first and the second column of the matrix

$\left(\begin{array}{l}p_{n+1} \\ z_{n+1}\end{array}\right)=\left(\begin{array}{ll}a_{11} & a_{12} \\ a_{21} & a_{22}\end{array}\right)\left(\begin{array}{l}p_{n} \\ z_{n}\end{array}\right)$. 


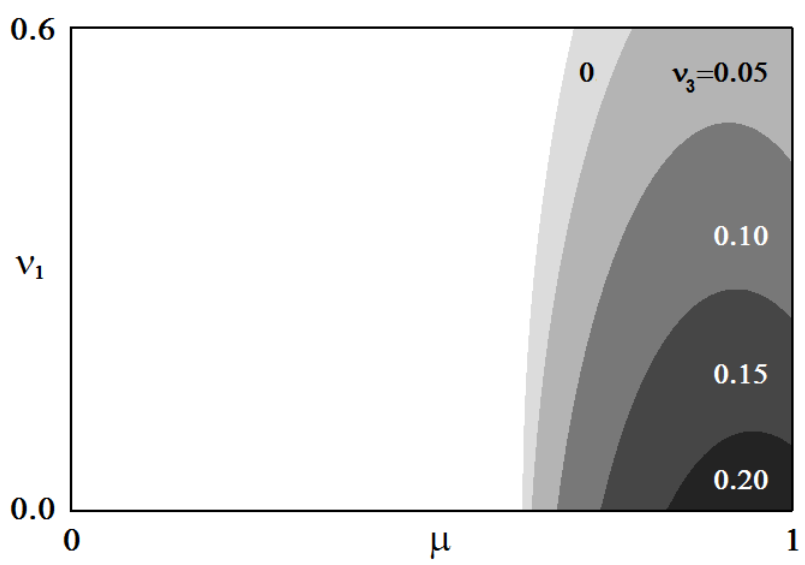

Fig. 4 Regions of parametric instability on the plane of parameters (a relative magnitude of the oscillating mass $\mu$ versus a coefficient of friction for the translational motion $\nu_{1}$ ) for $J=0.15, \varepsilon=1$. The values of the friction coefficient for the rotational component of motion $\nu_{3}$, which correspond to each region, are indicated by numbers.

The condition for instability is that there be at least one eigenvalue of the monodromy matrix whose absolute value exceeds 1 . Figure 4 shows zones of parametric instability for equations (13) on the plane of parameters $\mu$ and $\nu_{1}$ for several values of $\nu_{3}$ (the coefficient of friction of the rotational component of motion), which are indicated by numbers. We note that, in contrast to the mechanism considered in the previous section, acceleration in this case is achieved only when the relative magnitude of the oscillating mass is large enough. Also, when the parameters fit the zone of instability, acceleration to arbitrarily large values of momentum takes place despite the energy losses due to dissipation (friction). This is obvious from the linear nature of equations (24) (see also Fig. 5a). It should be noted that such parametric acceleration is accompanied by an unlimited increase in the oscillation of the reaction force, which determines the motion of the sleigh and is given in the normalized form by the expression (11). (The last term in this case vanishes.) In Fig.6 the panel (a) shows the dependence of the reaction force versus time as obtained in the numerical integration of the equations.

However, if the line of oscillations of the moving mass is displaced from the center of mass, it leads to saturation of the parametric instability, where the trajectory of the reduced equations evolves to an attractor. This is illustrated by diagrams (b) and (c) of Fig. 5, in which a chaotic attractor is approached. The degree of acceleration can be characterized by the root-meansquare value of the longitudinal momentum on the attractor. This value is bounded, but increases as the parameters approach the degenerate situation of linearity of equations (24). Now the oscillations of the reaction force appear also to be bounded, which is illustrated by the panel (b) in Fig.6.

Chaotic dynamics on the attractor of the reduced system corresponds to motion of the sleigh in the laboratory reference frame in the form of a random twodimensional walk of diffusion type, as is illustrated in Fig. 7.

Diagram (a) visualizes the results of numerical integration of equations for coordinates (10) together with the reduced equations (13) and shows how the trajectory of the sleigh looks like when the given parameters correspond to Fig.5c. The sleigh is started from the origin of coordinates $x=0, y=0$ with zero momentum $p$ and moment $q$ and with a zero initial angle of rotation $\varphi$. The motion is tracked up to the instant where the distance from the origin exceeds the given value $r_{\max }=100$. Further calculations continue with current values of $p, q$ and $\varphi$, but with a start again from the origin. Thus, the figure shows several successive fragments of the same trajectory, to which the orbit (approaching the chaotic attractor) of the reduced equations in Fig. $5 \mathrm{c}$ corresponds.

It can be seen that the motion observed in the case at hand is a two-dimensional random walk without a preferred direction, with the loss of memory of the initial sleigh orientation for large time scales [35, 36, 37. In this situation, the distribution of distances from the starting point to the end point of the trajectory segment travelled over a fixed number of oscillations of the internal mass $N$ (the number of iterations of the Poincaré map) must asymptotically tend to the Rayleigh distribution, and the distribution of azimuth angles, to a uniform distribution in the interval $[0,2 \pi)$.

Diagrams (b) and (c) show cumulative distributions for the distances $r=\sqrt{\left(x_{(k+1) N}-x_{k N}\right)^{2}+\left(y_{(k+1) N}-y_{k N}\right)^{2}}$ and the angles $\theta=\arg \left[\left(x_{(k+1) N}-x_{k N}\right)+i\left(y_{(k+1) N}-\right.\right.$ $\left.\left.y_{k N}\right)\right]$. The solid lines correspond to numerical results for a specified number of periods, $N=1000$. The set whose elements are fragments of the above-mentioned reference trajectory, which are labeled with index $k=$ $1 \ldots 10^{4}$, plays the role of the sample space. The dots in panel (a) correspond to the Rayleigh distribution $F(r)=1-e^{-r^{2} / 2 \sigma^{2}}$, where $2 \sigma^{2}$ is the sum of dispersions of random values $x_{(k+1) N}-x_{k N}$ and $y_{(k+1) N}-y_{k N}$, which have been obtained as statistical estimates from sampling; in the case at hand we have $\sigma^{2} \approx 5.3 \cdot 10^{5}$.

Generally speaking, attractor of the reduced system, which is approached under the conditions of a bounded parametric acceleration, can be chaotic or regular. Figure 8 shows two charts, plotted using different techniques, on the parameter plane (the same as in Fig. 4, where the regions of parametric instability were represented). The left panel presents a chart of regimes, 

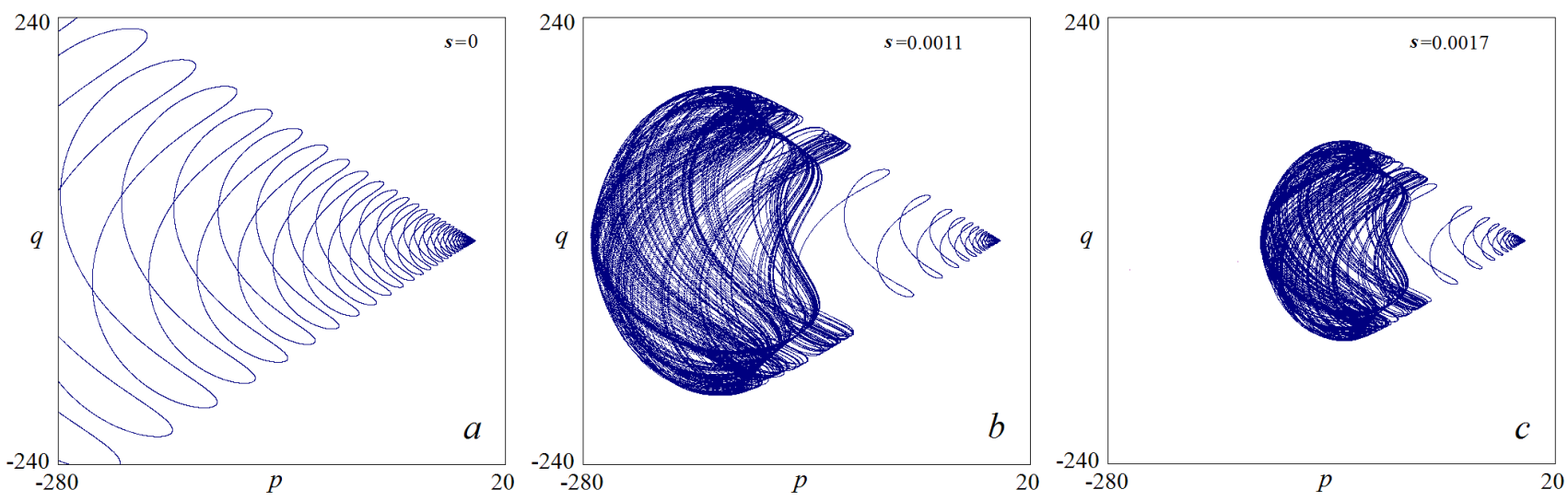

Fig. 5 Unbounded acceleration of the sleigh in a situation where the oscillating mass is located on the line passing through the center of mass (a) and bounded acceleration where the trajectory approaches a chaotic attractor (b), (c) with displacements from this line, according to the values of $s$ indicated in the figure. The center of mass of the platform coincides with the position of the knife edge, $a=0$. The other parameters are: $I_{0}=0.15, \mu=0.85, \nu_{1}=0.35, \nu_{3}=0.1, \varepsilon=1$.
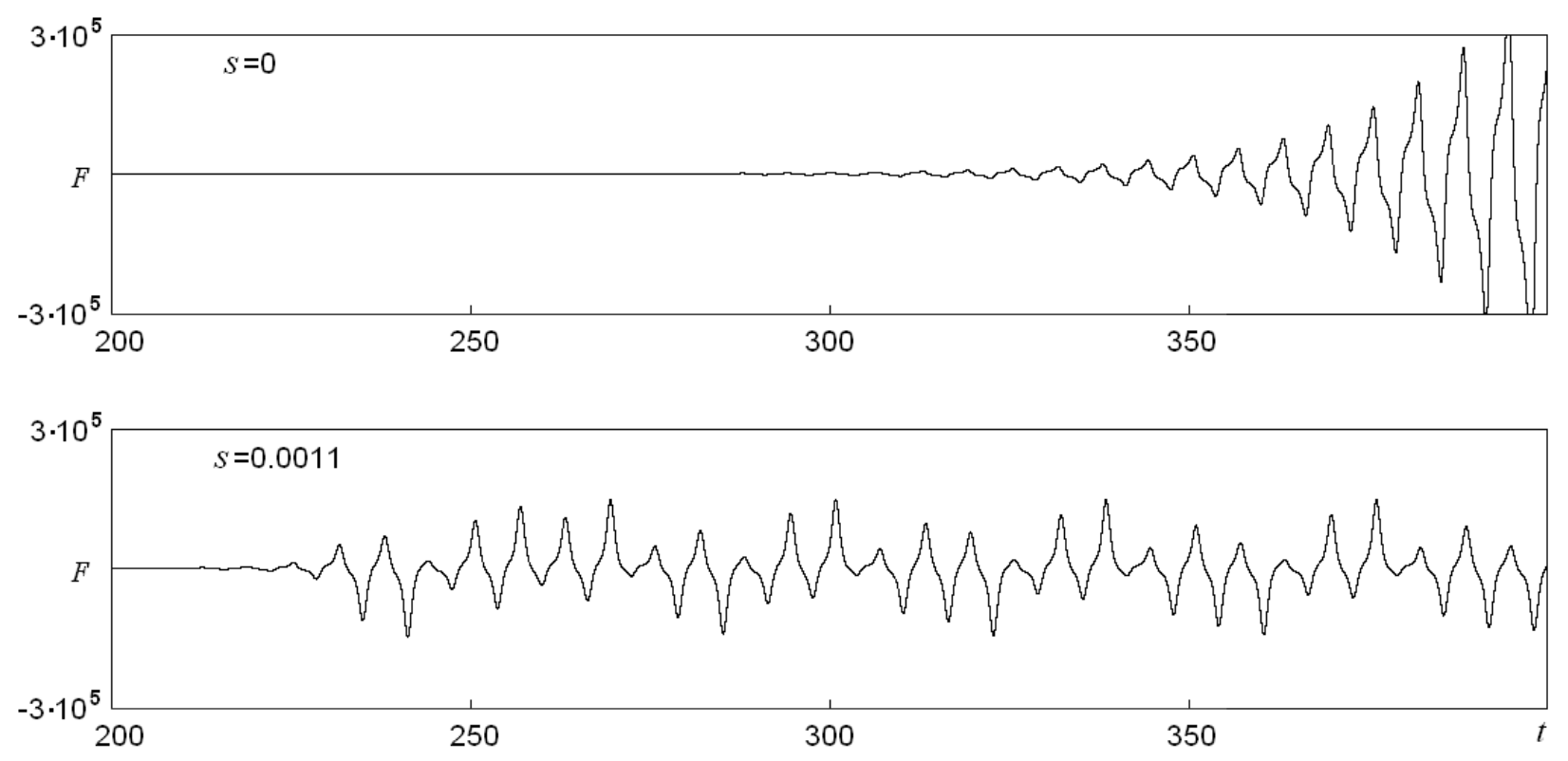

Fig. 6 Dependence of the normalized reaction force $F=\lambda / m \Omega^{2}$ acting at the location of the non-holonomic constraint versus time in the situation of unlimited acceleration at $s=0$ (a), and in the case of bounded acceleration with approach to the chaotic attractor in the phase space of the reduced equations at $s=0.0011$ (b). The center of mass of the platform coincides with the position of the knife-edge, $a=0$. Other parameters are $I_{0}=0.15, \mu=0.85, \nu_{1}=0.35, \nu_{3}=0.1, \varepsilon=1$.

where periodic ones are indicated with colors, and white denotes the region of chaos in accordance with the legend shown on the left. The right panel shows a chart of the same part of the parameter plane (plotted by analogy with a geographic map), where the colors code levels of the largest Lyapunov exponent: the levels below zero are indicated in blue, and the levels above zero are shown in yellow, red and brown, see the legend on the right of the chart.

Figure 9 contains a bifurcation diagram showing values of the dynamical variable $q$ (dimensionless angular momentum), which correspond to the attractor of the Poincaré map, versus the parameter of relative magni- tude of the oscillating mass $\mu$, and a graph of the largest Lyapunov exponent in the same interval of the parameter. On the parameter plane of Fig. 8, these diagrams correspond to the horizontal midline for $\nu_{1}=0.35$. Figure 10 depicts the attractors of the system with continuous time at representative points on the axis of $\mu$. These attractors are shown in light blue, and the dots corresponding to the stroboscopic Poincaré section are shown in red. Each panel contains an indication of the Lyapunov exponents for the Poincaré map which have been found numerically for the corresponding attractor.

When one moves along the axis of parameter $\mu$ from left to right, one first observes an attractor in the form 

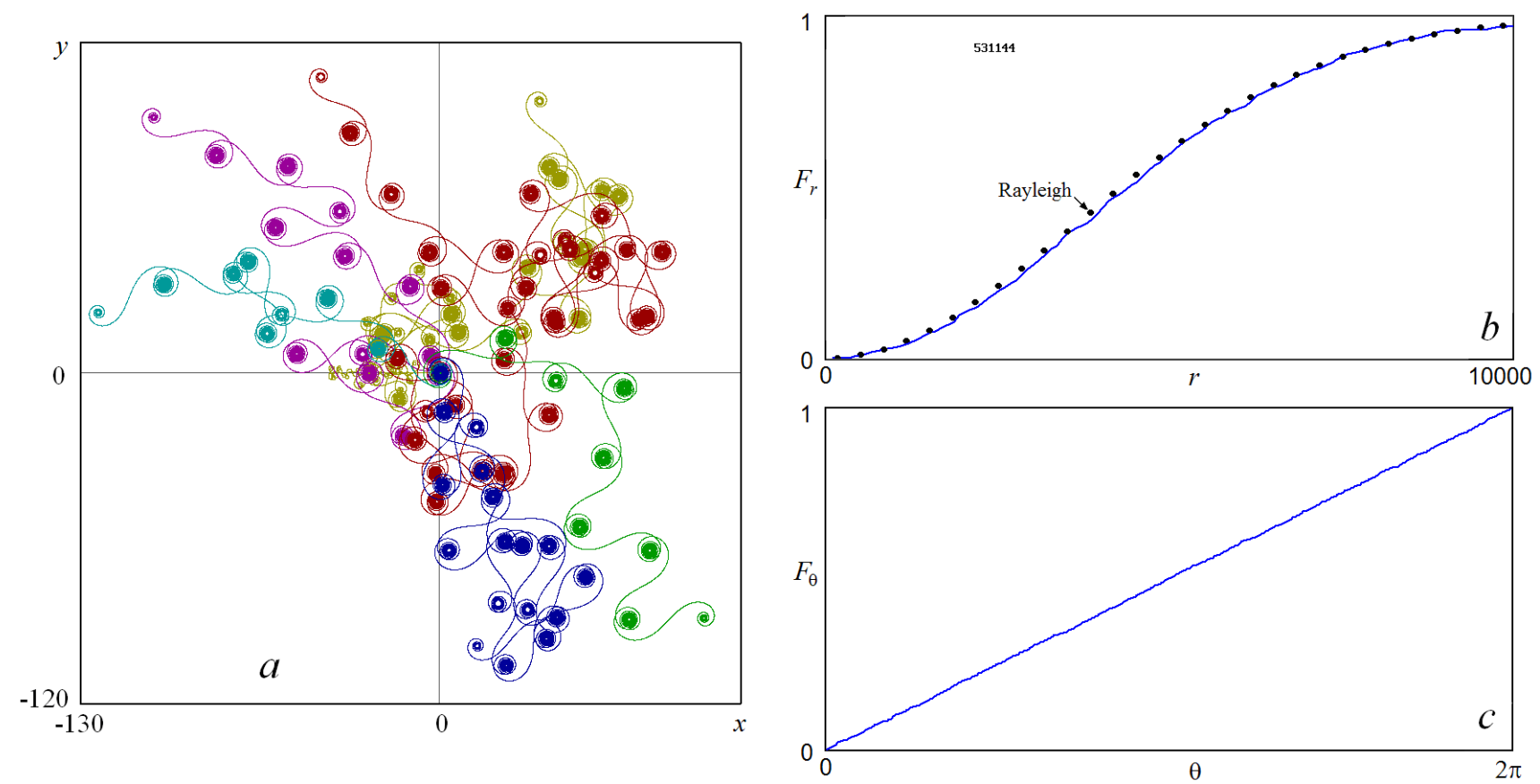

Fig. 7 Family of fragments of the trajectory of the sleigh in the laboratory reference frame, visualized as described in the text (a) and cumulative functions of distribution for the distances travelled (b) and for the azimuth angles (c), which were obtained for $10^{3}$ iterations of the Poincaré map. The data presented in diagram (b) are compared with the Rayleigh distribution (dots), and diagram (c) is indicative of a uniform distribution of the angles
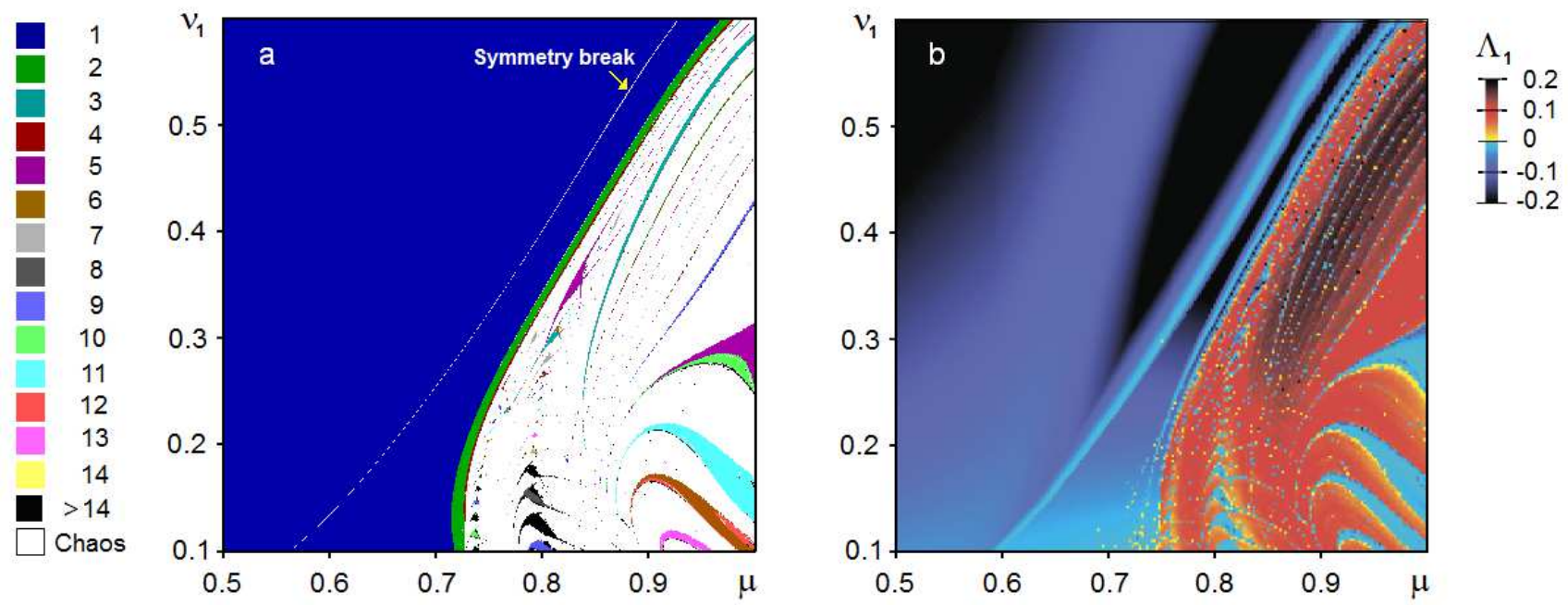

Fig. 8 Chart of periodic and chaotic regimes on the plane of parameters $\left(\mu, \nu_{1}\right)$ (a) and chart of Lyapunov exponents (b). The other parameters are: $I_{0}=0.15, \nu_{3}=0.1, \varepsilon=1, a=0, s=0.1$

of a symmetric loop, which corresponds to an attractive fixed point of the Poincaré map (panel $(\mathrm{a})$ ). Then one observes a symmetry-loss bifurcation $(\mu \approx 0.765)$, followed by a cascade of period-doubling bifurcations (panels (b) and (c)), and a transition to chaos. The resulting chaotic attractor is first asymmetric (d), so that in the phase space it coexists with a symmetric attractor resulting from the substitution (10). As the parameter $\mu$ grows, the attractor and its partner merge to form a single symmetric attractor (e). Such symmet- ric attractors can be seen in panels (g) and (i); on the other hand, panel (h) demonstrates a chaotic asymmetric attractor. The region of chaos is interspersed with periodicity windows, which look like light-colored vertical strips in the bifurcation diagram and like dips into a negative region in the graph of Lyapunov exponent. The attractor shown in panel (f) just corresponds to one of the regularity windows. 

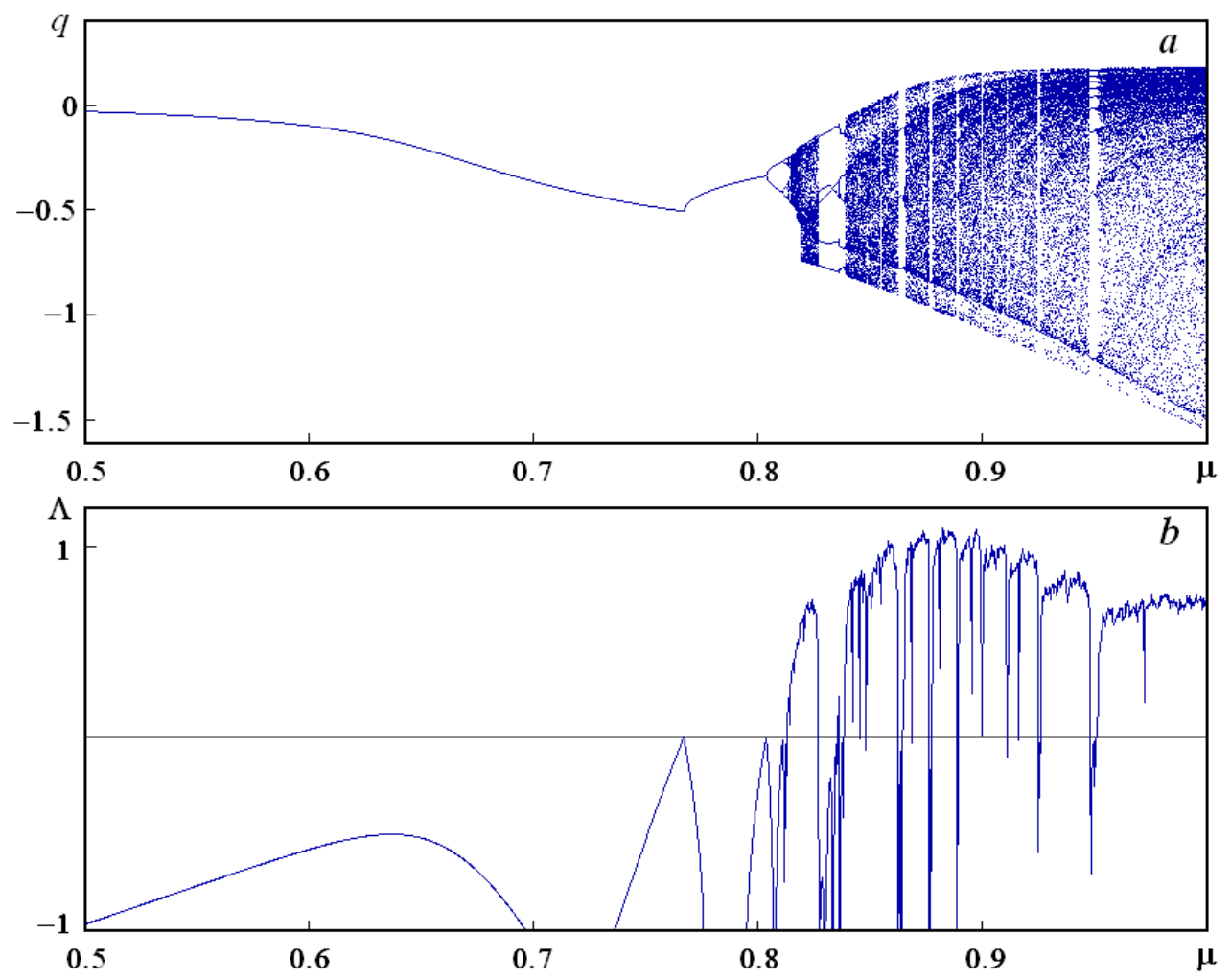

Fig. 9 The bifurcation diagram showing the dynamical variable $q$ on attractor of the Poincaré map versus parameter $\mu$ (a), and the plot of the largest Lyapunov exponent (b). The other parameters are: $I_{0}=0.15, \nu_{3}=0.1, \varepsilon=1, a=0, s=0.1$

\section{Comparison of chaotic dynamics with and without friction}

The object we consider here, namely, the Chaplygin sleigh with an oscillating internal mass, in the absence of friction is an example of behavior specific to nonholonomic systems with complex dynamics, which occurs due to invariance under time reversal. This implies a possibility of coexistence in the phase space of objects typical for conservative systems (regions occupied by closed invariant curves similar to KAM tori and regions of chaos - "chaotic seas") and typical for dissipative systems (regular and chaotic attractors). Due to invariance under time reversal, each attractor corresponds to a symmetric partner coexisting with it in the phase space - a repeller to which the phase trajectories are attracted when the dynamics is tracked in backward time. When friction is incorporated, these features, specific to reversible dynamics, degrade, and the behavior of the system acquires features typical of usual dissipative dynamics. In the concluding section of this paper we discuss the corresponding change in the dynamical behavior of our system, and the effect it has on the properties of the observed attractors.
In the absence of friction, when $\nu_{1}=0, \nu_{3}=0$, equations (7)-(8) take the form

$$
\begin{aligned}
& \dot{p}=(D w+\mu \varepsilon \cos \tau) w, \\
& \dot{q}=-(D w+\mu \varepsilon \cos \tau) u, \\
& u-w \mu \varepsilon \sin \tau=p, \\
& -u \mu \varepsilon \sin \tau+\left(J+\mu \varepsilon^{2} \sin ^{2} \tau\right) w=q-\mu s \varepsilon \cos \tau,
\end{aligned}
$$

and in this case, along with the symmetry (9), invariance under time reversal takes place. This corresponds to the involution

$p \rightarrow-p, q \rightarrow q, \tau \rightarrow-\tau, u \rightarrow-u, w \rightarrow w$.

Thus, if the system has an attractor, then a repeller symmetric relative to the above involution will necessarily coexist with this attractor in the phase space.

The chart in Fig. 11 gives an idea of the parameter space structure of the reversible system: the Lyapunov exponent that determines the coloring of each pixel according to the legend was calculated for the corresponding parameters and identical initial conditions $(p=5$, $q=0, \tau=0)$. At the periphery one can see phase portraits of the stroboscopic Poincaré map at representative points (a), (b) and (c), which were plotted in each case for several sets of initial conditions.

The structure of the phase plane shown in diagrams (a) and (b) is similar to that for conservative Hamilto- 

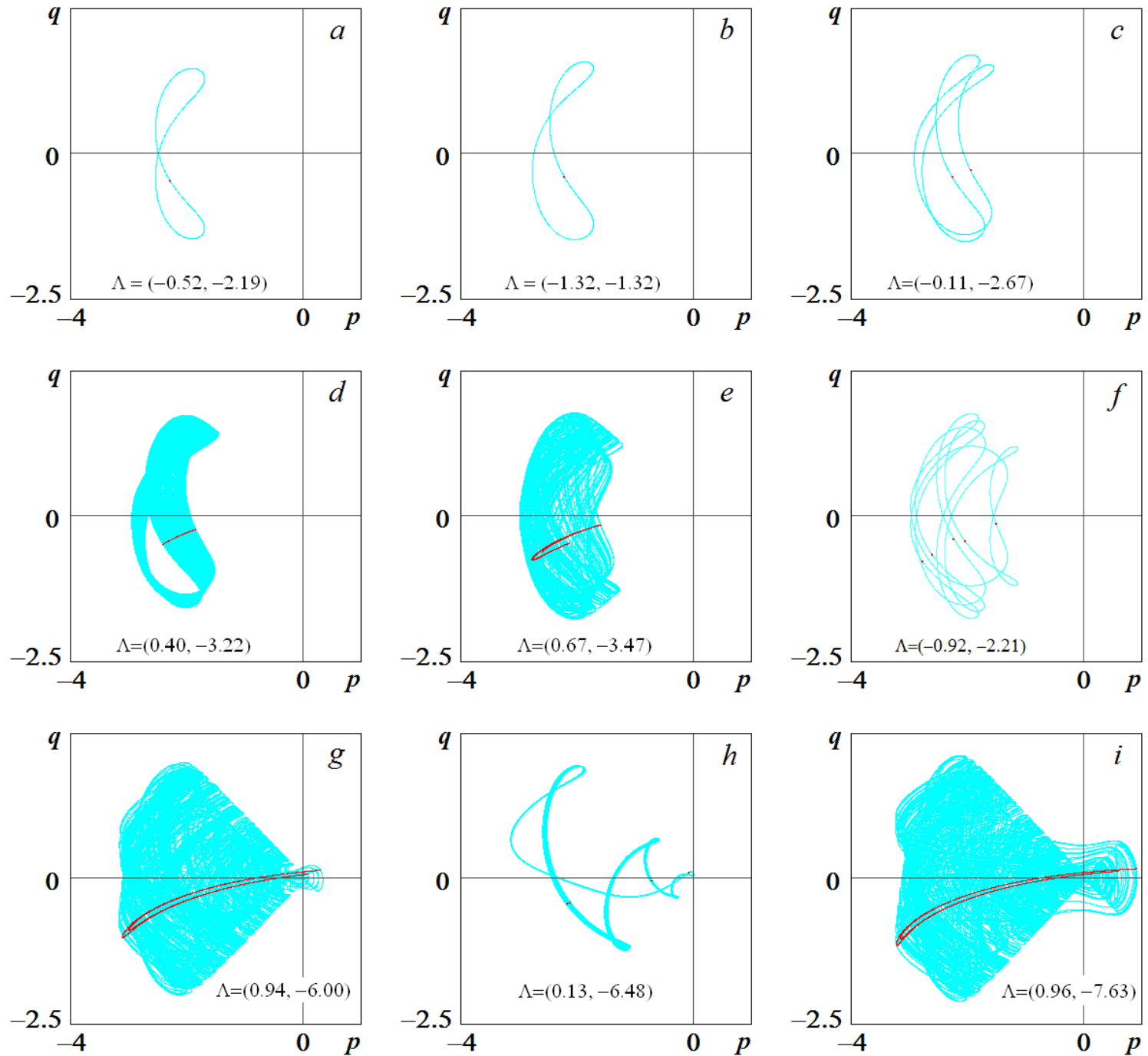

Fig. 10 Attractors of the reduced equations for parameters $I_{0}=0.15, \nu_{3}=0.1, \varepsilon=1, a=0, s=0.1$ and $\mu=0.75$ (a), 0.78 (b), $0.81(\mathrm{c}), 0.816(\mathrm{~d}), 0.825(\mathrm{e}), 0.83(\mathrm{f}), 0.89(\mathrm{~g}), 0.9(\mathrm{~h}), 0.92(\mathrm{i})$. Light-blue denotes trajectories in continuous time, and red indicates dots corresponding to the stroboscopic Poincaré section at time instants $t=2 \pi n$. The Lyapunov exponents of the stroboscopic map are indicated under the portraits of the attractors

nian systems with discrete time, namely, the regions occupied by closed invariant curves similar to KAM tori, and the chaotic sea regions. The latter should be treated as a sort of "fat attractors", since the positive and negative Lyapunov exponents for them differ in absolute value (see the values of the exponents for the Poincaré map which are indicated in the panels). At the same time, for motions on invariant curves the spectrum of Lyapunov exponents according to calculations consists of two zero exponents (with accuracy to calculation errors).

In diagram (c), one can see an attractor having a pronounced transverse Cantor-like structure of filaments similar to attractors in dissipative systems, while one observes no regions occupied by invariant curves.
We note that the estimate of the dimension from the Kaplan-Yorke formula $D_{K Y}=1+\Lambda_{1} /\left|\Lambda_{2}\right|$ for the "fat" attractors (a) and (b) gives 1.54 and 1.84, i.e., the dimension is rather large. For the "thin" quasi-dissipative attractor (c) the dimension is only 1.17 .

Figure 12 presents a comparison of the Lyapunov charts for a system without friction $\nu_{1}=0$ (a) and for friction coefficients $\nu_{1}=0.05$ (b) and 0.1 (c). In all cases it is assumed that $\nu_{3}=0$. As can be seen, introducing even a small dissipation leads to "washing-out" of the region of existence of chaos associated with fat attractors. These are now occupied by regimes of regular dynamics. In contrast, there is a relatively small influence of dissipation on chaotic attractors of the type shown in Fig. 11c, which reside in the black region in the right lower part of the chart. The action of friction 


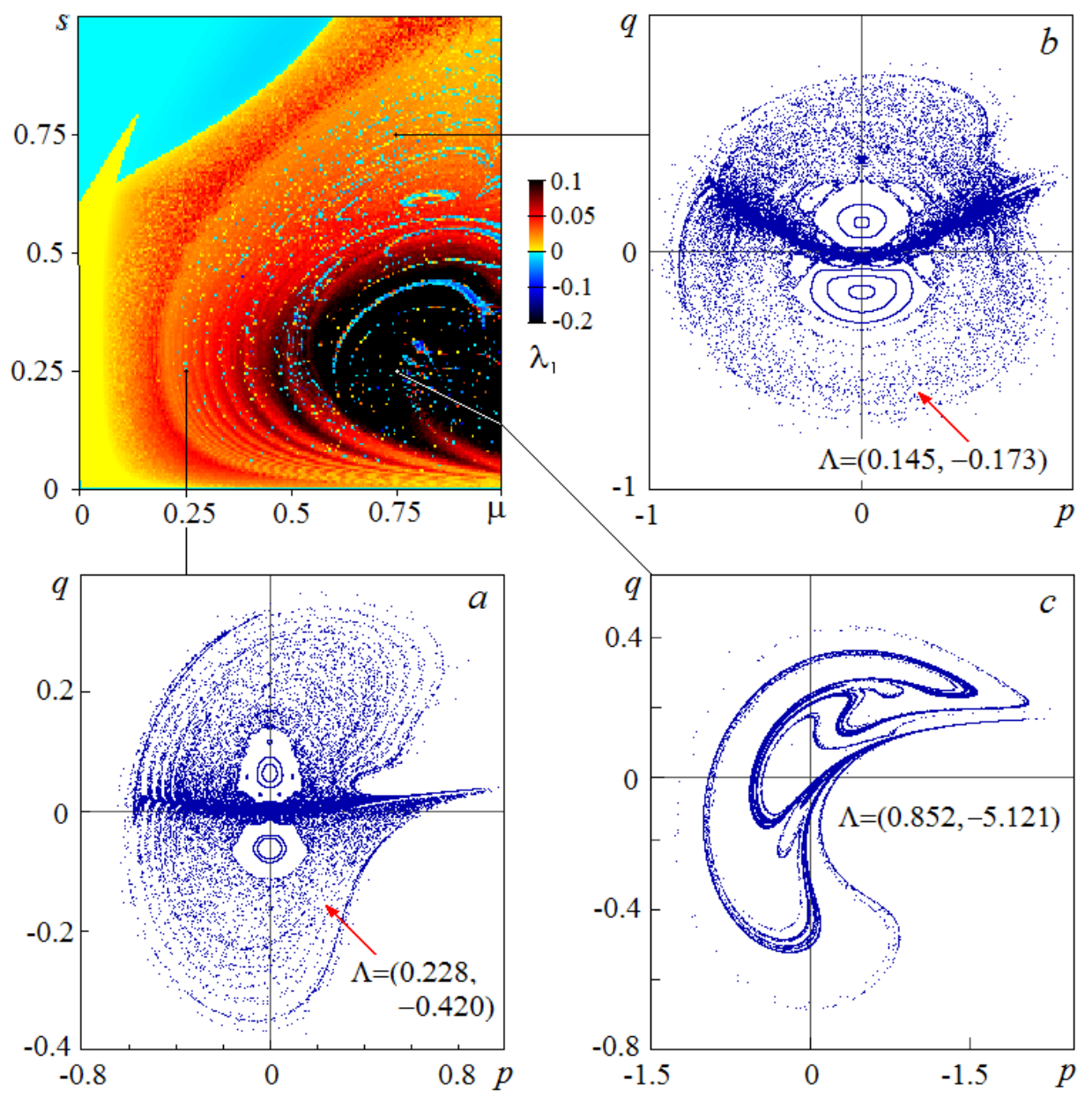

Fig. 11 Chart of Lyapunov exponents on the parameter plane for a system without dissipation and portraits illustrating the structure of the phase plane in the regions where it has a similarity with conservative dynamics (a), (b) and with dissipative dynamics (c). The values of the parameters are $\mu=0.25, s=0.25$ (a), $\mu=0.25, s=0.75$ (b), $\mu=0.75, s=0.25$ (c); the values of the other parameters are $\varepsilon=1, a=0.5, I_{0}=0.05, \nu_{1}=0, \nu_{3}=0$. The Lyapunov exponents of the chaotic dynamics for the Poincaré map are indicated in panels (a)-(c)
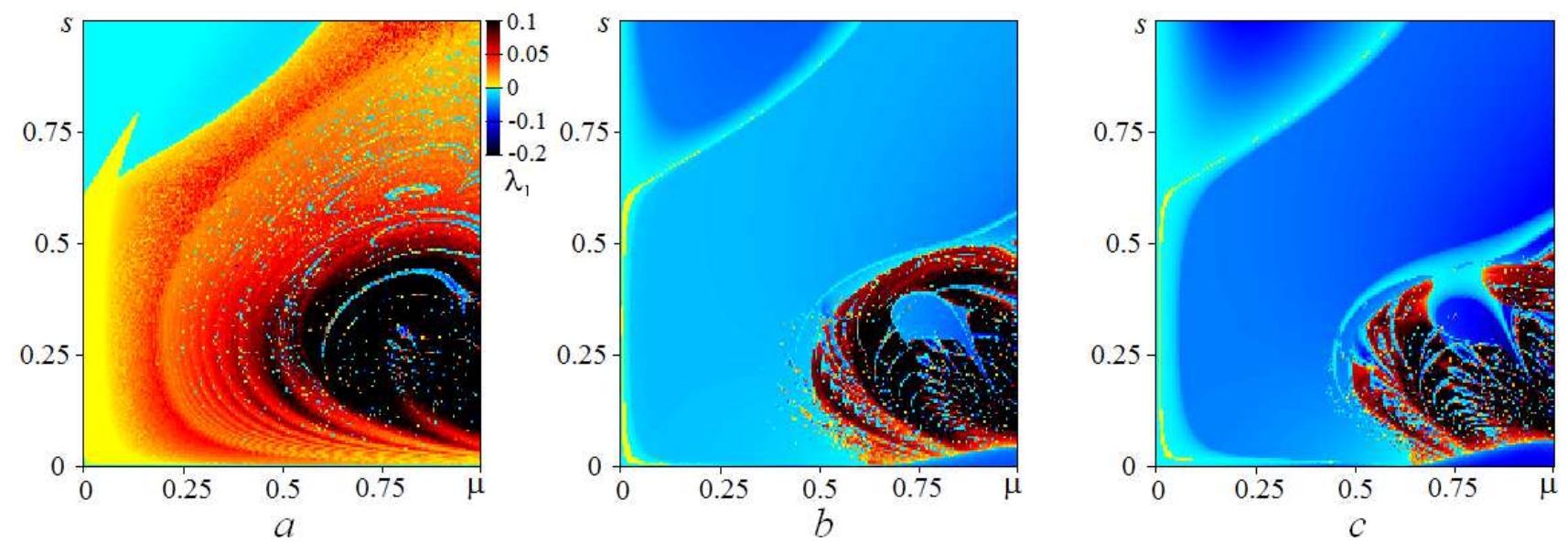

Fig. 12 Lyapunov charts for a system without friction $\nu_{1}=0$ (a) and with friction coefficients $\nu_{1}=0.05$ (b) and 0.1 (c). The other parameters are: $\varepsilon=1, a=0.5, I_{0}=0.05, \nu_{3}=0$ 

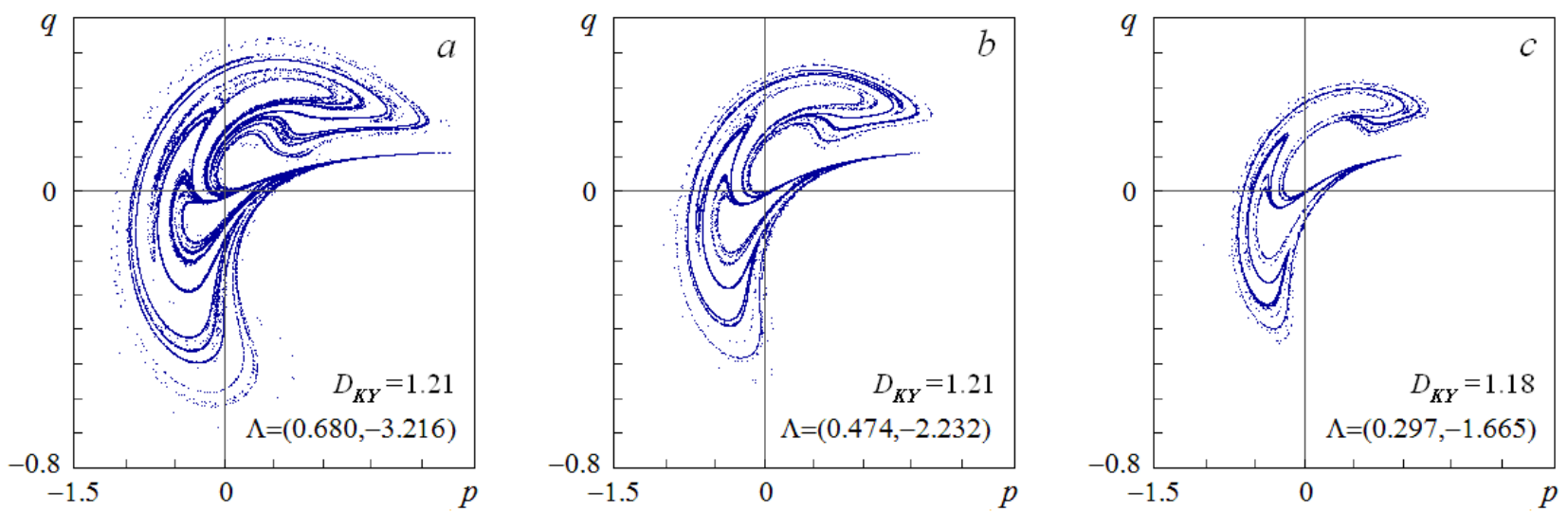

Fig. 13 Portraits of attractors of the stroboscopic Poincaré map for a system without friction $\nu_{1}=0$ (a) and for that with friction coefficients $\nu_{1}=0.05$ (b) and 0.1 (c). The other parameters are: $\varepsilon=1, a=0.5, I_{0}=0.05, s=0.2, \mu=0.6, \nu_{3}=0$. In the lower parts of the panels, Lyapunov exponents for the attractor of the Poincaré map are indicated and the estimates of the Kaplan-Yorke dimension are given

reduces to some changes in the quantitative characteristics (Lyapunov exponents), however, the general structure of the attractors remains approximately the same, moreover, the Kaplan-Yorke dimension does almost not change over the above-mentioned range of values of the dissipation parameter. The aforesaid is illustrated by portraits of attractors in the stroboscopic section in Fig. 13, which have been constructed for $\nu_{1}=0$ (a), 0.05 (b) and 0.1 (c). In the lower parts of the panels, Lyapunov exponents for the Poincaré map are indicated and estimates of the Kaplan-York dimension are given.

Thus, it may be concluded that chaotic motions of a nonholonomic system are divided into two classes and occur in specific parameter regions.

One kind of chaos is associated with fat attractors, which can be compared with chaotic seas of conservative Hamiltonian systems. Like chaotic seas, fat attractors coexist with phase space regions which are filled with invariant curves and correspond to quasi-periodic dynamics. In the chart of Fig. 11, such dynamics correspond to the orange-red region.

On the other hand, the chaos related to attractors of the second kind is analogous to dissipative systems where the attractor exhibits a pronounced transverse split structure of filaments. This corresponds to the dark region in the right lower part of the chart in Fig. 11 (upper left panel).

The difference between both types of attractors lays in the presence or absence of a structure in the form of split filaments and thus in dimension properties - for fat attractors the dimension is much higher. The difference also lays in the degree of sensitivity to incorporation of friction: in the region where fat attractors manifest themselves, chaos is effectively washed out when dissipation is incorporated, and is replaced with regular motions. This does not happen to attractors of the second kind, and chaos survives in the presence of dissipation, at least as long as the latter does not exceed some moderate level. Thus, in the presence of friction, the specific properties of the nonholonomic system become less pronounced, and the behavior becomes to a greater extent similar to that typical of dissipative systems with complex dynamics.

\section{Conclusion}

The motion of the Chaplygin sleigh in the presence of an oscillating internal mass in the presence of viscous friction is considered. It is assumed that a material point oscillates relative to the main platform when moving in a straight line perpendicularly to the axis passing through the center of mass of the platform and through the point of application of the nonholonomic constraint in the direction coinciding with that of the knife edge which ensures the nonholonomic constraint. Equations governing the dynamics of the system are formulated. The time evolution of momentum and angular momentum is governed by a reduced system of equations (decoupled from the other equations pertaining to the configuration space), and during the motion of the internal mass their change is determined by a two-dimensional map. The other configuration variables, including the coordinates in the laboratory reference frame and the angle of rotation of the sleigh, are expressed in terms of the momentum variables using quadratures.

The results presented in this paper can be extended to wheeled vehicles, since the nonholonomic constraint corresponding to the Chaplygin sleigh is equivalent to that which is introduced by replacing the knife edge by a wheel pair [18] with the other supports sliding freely. 
For the motion of the Chaplygin sleigh in the presence of weak friction, two mechanisms of acceleration due to oscillations of the internal mass have been found and investigated. Under small oscillations of the internal mass the sleigh may exhibit acceleration of motion that is on average rectilinear; in the absence of friction the acceleration is unbounded, while in the presence of friction it becomes modified so that the velocity reached is stabilized at a fixed level - the smaller the friction, the higher the velocity. The other mechanism is due to the effect of parametric excitation of oscillations, when the mass which executes oscillations is comparable in magnitude to the mass of the main platform. In this case, the presence of friction is necessary for acceleration. In the simplest case of motion of the internal mass in a straight line passing through the center of mass, the problem reduces to a linear equation with periodic coefficients, and the increase in parametric oscillations is unbounded, as in the Mathieu equation. As is usually the case under parametric oscillations, linear dissipation does not lead to saturation; the saturation occurs only in the presence of nonlinear effects. In our case, the parametric instability and the resulting acceleration of the sleigh are bounded if the line of oscillations of the moving mass is displaced from the center of mass. The sustained regime of motion is associated with the attractor of the reduced system of equations, which in many cases turns out to be chaotic; accordingly, the motion of the sleigh is also chaotic and similar to a diffusion process.

Also, a qualitative analysis has been made of the dynamics in the absence of friction, when the model under consideration provides an example of complex dynamics which is specific to nonholonomic systems and is due to invariance under time reversal, and a modification of this behavior with dissipation has been discussed. In particular, it is shown that the incorporation of dissipation drastically changes the dynamics in parameter regions where the model without friction demonstrates coexistence of quasi-conservative types of behavior, namely, phase space regions filled with invariant curves and chaos corresponding to fat attractors. On the other hand, in the parameter region where there are attractors similar in nature to attractors of dissipative systems (fractal transverse structure of filaments), the introduction of dissipation leads only to certain quantitative changes in the spectrum of Lyapunov exponents, without a considerable change in the dimension of the attractors.

The existence of chaotic attractors exhibited by the Chaplygin sleigh with a moving internal mass makes it possible to apply chaos control methods [38 for organizing the motion in a specific direction by changing the characteristics of oscillatory motion of the internal mass under conditions of diffusion type sleigh dynamics. Since chaos is sensitive to small perturbations, control can be performed by an arbitrarily small targeted action.

The results obtained here supplement the methods (considered in other publications) for controlling the motions of mobile mechanical systems whose characteristics can be changed by regulating the parameters, including the coefficient of intensity of impulses of external forces [20,21], by switching the nonholonomic constraint to different locations 23,24, and by changing the amplitude of oscillations of the internal mass or its position [25,26].

Acknowledgements This work was supported by grant No. 15-12-20035 of the Russian Science Foundation.

\section{References}

1. Goldstein, H., Poole, Ch.P. Jr., Safko, J.L.: Classical Mechanics, 3rd ed., Addison-Wesley, Boston, Mass. (2001)

2. Gantmacher, F.R.: Lectures in Analytical Mechanics. Mir, Moscow (1975).

3. Neimark, Ju.I., Fufaev, N.A.: Dynamics of Nonholonomic Systems, Translations of Mathematical Monographs, Vol. 33. American Mathematical Society, Providence (2004).

4. Borisov, A.V., Mamaev, I.S., Bizyaev, I.A.: Historical and critical review of the development of nonholonomic mechanics: the classical period. Regular and Chaotic Dynamics 21(4), 455-476 (2016).

5. Borisov, A.V., Mamaev, I.S.: Rigid body dynamics. RCD, Izhevsk (2001) (In Russian.)

6. Bloch, A.M.: Nonholonomic mechanics and control. Springer (2015)

7. Borisov, A.V., Mamaev, I.S., Bizyaev, I.A.: Historical and Critical Review of the Development of Nonholonomic Mechanics: the Classical Period. Regular and Chaotic Dynamics 21(4), 455-476 (2016)

8. Li, Z., Canny, J.F. (ed.): Nonholonomic motion planning. Springer Science \& Business Media (2012)

9. Fukao, T., Nakagawa, H., Adachi, N.: Adaptive tracking control of a nonholonomic mobile robot. IEEE transactions on Robotics and Automation 16(5), 609-615 (2000)

10. Alves, J., Dias, J.: Design and control of a spherical mobile robot. Proceedings of the Institution of Mechanical Engineers, Part I: Journal of Systems and Control Engineering 217(6), 457-467 (2003)

11. Borisov, A.V., Kilin, A.A., Mamaev, I.S.: How to control Chaplygin's sphere using rotors. Regular and Chaotic Dynamics 17(3), 258-272 (2012)

12. Borisov, A.V., Mamaev, I.S.: Conservation laws, hierarchy of dynamics and explicit integration of nonholonomic systems. Regular and Chaotic Dynamics 13(5), 443-490 (2008)

13. Borisov, A.V., Mamaev, I.S.: Strange attractors in rattleback dynamics. Physics-Uspekhi 46 (4), 393-403 (2003)

14. Borisov, A.V., Kazakov, A.O., Kuznetsov, S.P.: Nonlinear dynamics of the rattleback: a nonholonomic model. Physics-Uspekhi 57(5), 453-460 (2014). 
15. Borisov, A.V., Jalnine, A.Yu., Kuznetsov, S.P., Sataev, I.R., Sedova, J.V.: Dynamical phenomena occurring due to phase volume compression in nonholonomic model of the rattleback. Regular and Chaotic Dynamics, 17(6), 512-532 (2012)

16. Chaplygin, S.A.: On the Theory of Motion of Nonholonomic Systems. The Reducing-Multiplier Theorem. Regular and Chaotic Dynamics 13(4), 369376 (2008); see also: Mat. Sb., 28(2), 303314 (1912)

17. Caratheodory, C.: Der Schlitten. Z. Angew. Math. Mech. 13(2), 7176 (1933).

18. Borisov, A.V., Kilin, A.A., Mamaev, I.S.: On the Hadamard - Hamel Problem and the Dynamics of Wheeled Vehicles. Regular and Chaotic Dynamics 20(6), 752766 (2015)

19. Borisov, A.V., Mamaev, I.S.: The dynamics of a Chaplygin sleigh. Journal of Applied Mathematics and Mechanics 73(2), 156161 (2009)

20. Borisov, A.V., Kuznetsov, S.P.: Regular and Chaotic Motions of a Chaplygin Sleigh under Periodic Pulsed Torque Impacts. Regular and Chaotic Dynamics 21(7-8) 792803 (2016)

21. Tallapragada, P., Fedonyuk, V.: Steering a Chaplygin sleigh using periodic impulses. Journal of Computational and Nonlinear Dynamics, 12(5), 054501 (2017).

22. Fedonyuk, V., Tallapragada, P.: The dynamics of a two link Chaplygin sleigh driven by an internal momentum wheel. In American Control Conference (ACC), IEEE, 2171-2175 (2017)

23. Kuznetsov, S.P.: Regular and chaotic motions of the Chaplygin sleigh with periodically switched location of nonholonomic constraint. Europhysics Letters, 118(1), 10007 (2017)

24. Kuznetsov, S.P.: Regular and Chaotic Dynamics of a Chaplygin Sleigh due to Periodic Switch of the Nonholonomic Constraint. Regular and Chaotic Dynamics 23(2), 178192 (2018)

25. Bizyaev, I.A., Borisov, A.V., Mamaev, I.S.: The Chaplygin Sleigh with Parametric Excitation: Chaotic Dynamics and Nonholonomic Acceleration. Regular and Chaotic Dynamics 22(8), 957977 (2017)

26. Bizyaev, I.A., Borisov, A.V., Kuznetsov, S.P.: Chaplygin sleigh with periodically oscillating internal mass. Europhysics Letters, 119(6), 60008 (2017).

27. Fedonyuk, V., Tallapragada, P.: Sinusoidal control and limit cycle analysis of the dissipative Chaplygin sleigh. Nonlinear Dynamics (2018) https://doi.org/10.1007/s11071-018-4230-1

28. Jung, P., Marchegiani, G., Marchesoni, F.: Nonholonomic Diffusion of a Stochastic Sled. Physical Review E 93(1), 012606 (2016).

29. Halme, A., Schonberg, T., Wang, Y.: Motion control of a spherical mobile robot. Advanced Motion Control. Proceedings., 4th International Workshop, 259-264. IEEE (1996)

30. Borisov A.V., Kilin A.A., Mamaev I.S.: How to control Chaplygin's sphere using rotors. Regular and Chaotic Dynamics 17(3), 258-272 (2012)

31. Pollard, B., Tallapragada, P.: An aquatic robot propelled by an internal rotor. IEEE/ASME Transactions on Mechatronics 22(2), 931-939 (2017)

32. Fermi, E.: On the origin of the cosmic radiation. Physical Review 75(8), 1169-1174 (1949)

33. Lichtenberg, A.J., Lieberman, M.A., Cohen, R.H.: Fermi acceleration revisited. Physica D: Nonlinear Phenomena 1(3), 291-305 (1980)
34. Yudovich, V.I.: The dynamics of vibrations in systems with constraints. Physics-Doklady 42, 322-325 (1997)

35. Feller, W.: An Introduction to Probability Theory and its Applications, vol.1, 3-d ed. Wiley (1968)

36. Rytov, S.M., Kravtsov, Y.A., Tatarskii, V.I.: Principles of Statistical Radiophysics. 1. Elements of Random Process Theory. Springer, 1987.

37. Cox, D.R., Miller, H.D.: The Theory of Stochastic Processes. CRC Press, 1973.

38. Schöll, E., Schuster, H.G. (ed.): Handbook of chaos control. John Wiley \& Sons (2008). 